\title{
APLICAÇÃO DO MÉTODO DE SEPARAÇÃO DE VARIÁVEIS AO PROBLEMA DA DINÂMICA DA MEMBRANA HETEROGÊNEA
}

\author{
Gonzaga, B. R; Loeffler C. F.
}

Programa de Pós-Graduação em Engenharia Mecânica, Universidade Federal do Espírito Santo, Vitória, ES, Brasil

\begin{abstract}
Resumo
A pesquisa envolvida no presente trabalho apresenta uma metodologia analítica de solução de um problema dinâmico não homogêneo através do Método de Separação de Variáveis. Esta iniciativa tem o objetivo de colaborar para o estabelecimento de um banco de soluções analíticas que possa servir para validar as respostas dos métodos numéricos quando aplicados em diferentes áreas no estudo de ondas. Isto porque verifica-se que a disponibilidade de soluções analíticas na área de propagações de ondas em meios heterogêneos é muito reduzido, necessitando assim de contribuições que possam qualificar com maior precisão os resultados de diversas técnicas numéricas. O Método de Separação de Variáveis permite solucionar alguns destes problemas de grande complexidade reduzindo o modelo diferencial parcial para um conjunto de equações diferenciais ordinárias para cada variável independente. Em razão da complexidade do presente problema, funções especiais aparecem na modelagem requerendo uma manipulação matemática sofisticada e certamente o auxílio de um programa computacional que resolva os algebrismo se integrais mais complexas.
\end{abstract}

Palavras-chaves: Método de Separação de Variáveis, Equação da Onda, Soluções Analíticas de Referência.

\section{Introdução}

As Equações Diferenciais Parciais (EDPs) modelam diversos problemas geométricos, físicos, químicos e biológicos de importância para engenharia e outras áreas. Em tais equações, grandezas de interesse são expressas por funções desconhecidas, que dependem de duas ou mais variáveis, geralmente o tempo e as coordenadas espaciais [1].

Desde os primórdios da Física Matemática, a Equação Escalar da Onda, também conhecida como Equação Acústica da Onda, foi alvo de estudos clássicos de diversos matemáticos, tais como Euler, Daniel Bermoulli e Lagrange, que partindo de problemas simples uniaxiais, desenvolveram técnicas para solução de casos bi e tridimensinais, resolvendo situações com dissipação, dispersão e propagação em meios infinitos, entre outras. Atualmente visando aprimorar modelos matemáticos baseados na propagação de ondas mecânicas para abordar problemas sismológicos, hidrogeológicos e de prospecção geofísica, o desenvolvimento de estudos relativos à propagação de ondas tem considerado também a heterogeneidade das propriedades constitutivas do meio, principalmente no contexto da sísmica de prospecção. Por outro lado, há várias décadas as pesquisas da engenharia e da física matemática se concentram em métodos aproximados para resolver as EDPs que, via de regra, se tornam extremamente complicadas quando as condições de contorno são gerais e os fenômenos físicos envolvem anisotropia, heterogeneidade e não linearidades diversas. Nestas condições mais arrojadas são muito poucas as soluções analíticas para as EDPs correspondentes.

No que se refere aos problemas da sísmica de prospecção, é notória a ausência de um número significativo de problemas teste possuem soluções analíticas em que o meio seja heterogêneo.

\section{Objetivo}

O objetivo deste trabalho é apresentar a solução de uma das etapas do Método de Separação de Variáveis (MSV), cuja característica essencial é substituir a EDP original por um conjunto de equações diferenciais ordinárias, sujeitas a condições iniciais e de contorno [2]. Em geral, as soluções são expressas em termos de soma de séries infinitas, tema que envolve conceitos expressos no estudo das Séries de Fourier [1]. 


\section{Modelo Matemático}

No caso deste trabalho, uma membrana quadrada, heterogênea, é fixada em três de suas arestas e submetida a um deslocamento dinâmico súbito. A figura (1) ilustra as características físicas e as condições de contorno admitidas.

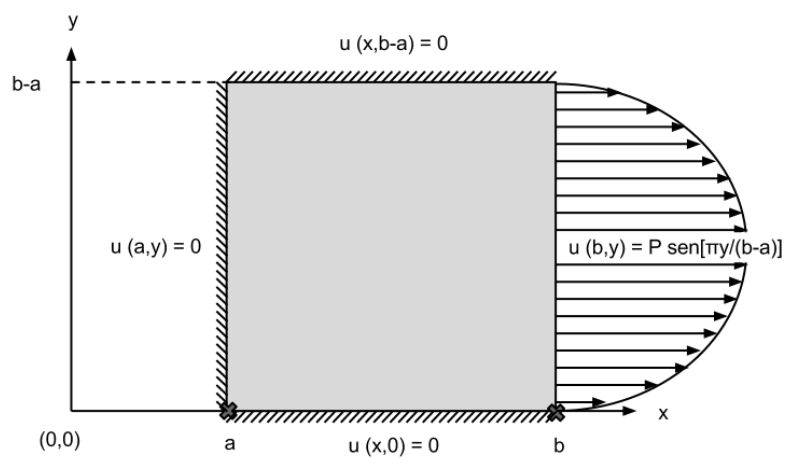

Figura 1: Características geométricas da membrana.

A abordagem doravante apresentada é válida também para outros tipos de condição de contorno, bem como outras formas de carregamento com diferente dependência no tempo. Ressalta-se, entretanto, que a escolha do sistema de coordenadas deve ser estratégica, para que a solução analítica não se apresente inviável. A EDP de governo, nas condições de não homogeneidade, é dada por [3]:

$$
\nabla \cdot[E(x) \nabla u(x, y)]=\rho \frac{\partial^{2} u(x, y)}{\partial t^{2}}
$$

Por simplicidade, a variação da propriedade física é admitida ocorrer unicamente na direção x, na forma:

$E(x)=\frac{E_{0} x}{a}$

Com esta última consideração, a Equação da Onda fica na forma:

$x \frac{\partial^{2} u}{\partial x^{2}}+a \frac{\partial u}{\partial x}+x \frac{\partial^{2} u}{\partial y^{2}}=\frac{\rho}{E_{o}} \frac{\partial^{2} u}{\partial t^{2}}$

As condições iniciais são dadas por:

$\dot{u}(x, y, 0)=0 ; u(x, y, 0)=0$

$E$ as condições de contorno, conforme se pode verificar da Fig. (1) são expressas por:

$$
\begin{aligned}
& u(b, y)=P_{0} \operatorname{sen}\left(\frac{\pi y}{L}\right) \\
& u(a, y)=u(x, 0)=u(x,(b-a))=0
\end{aligned}
$$

\section{Aplicação do MSV}

Devido haver uma condição de contorno não homogênea no presente problema, para que se possa usar o MSV faz-se necessário adaptá-la usando uma função auxiliar que a obedeça separadamente. Esta função auxiliar será denominada de $\phi(x, y)$. Então, tem-se:

$u(x, y, t)=\phi(x, y)+v(x, y, t)$

Onde:

$\phi(x, y)=\sum_{n=1}^{\infty} W_{n}(x) Z_{n}(y)$

$v(x, y, t)=\sum_{n=1}^{\infty} X_{n}(x) Y_{n}(y) T_{n}(t)$

O exame das condições de contorno estabelece uma relação entre as soluções $W_{n}(x)$ e $Z_{n}(y)$ de modo que resultam as seguintes equações pelo MSV:

$W_{n}^{\prime \prime}+\frac{1}{x} W_{n}^{\prime}-k_{n}^{2} W_{n}=0 \quad Z_{n}^{\prime}+k_{n}^{2} Z_{n}=0$

A solução de $Z_{n}(y)$ é simples, de modo que com a imposição das condições de contorno resulta:

$Z_{n}(y)=C_{n} \operatorname{sen}\left(\frac{n \pi y}{L}\right)$

Da mesma forma, a solução de $W_{n}(x)$ fornece:

$$
\begin{gathered}
W_{n}(x)=P_{0} G\left[\left(-\frac{B \operatorname{essel} Y\left[0,-i k_{1} a\right]}{B \operatorname{Bessel} J\left[0, i k_{1} a\right]}\right) \operatorname{Bessel}\left[0, i k_{1} x\right]+\right. \\
\left.\left.\left.+\operatorname{Bessel} Y\left[0,-i k_{1} x\right]\right)\right]\right]
\end{gathered}
$$

Onde G é uma constante conhecida, obtida pelas condições de contorno. Ressalta-se que as funções de Bessel presentes na solução dada pela Eq. (12) fornecem valores reais. Então, depois de aplicados os devidos valores, a função auxiliar se apresenta da seguinte forma [4]:

$$
\begin{aligned}
& \phi(x, y)=Q\left[\left[\left(-\frac{B \operatorname{Bssel} Y\left[0,-i \frac{\pi}{2}\right]}{B \operatorname{essel} J\left[0, i \frac{\pi}{2}\right]}\right) \text { Bessel }\left[0, i \frac{\pi}{2} x\right]+\right.\right. \\
& \left.\left.\left.+\quad B e s \operatorname{sel} Y\left[0,-i \frac{\pi}{2} x\right]\right)\right]\right] \operatorname{sen}\left(\frac{\pi y}{L}\right)
\end{aligned}
$$

Já para a solução dependente do tempo $v(x, y, t)$, a aplicação do MSV resulta em funções $Y_{n}(y)$ e $Z_{n}(t)$ simples, harmônicas; obtidas a partir da seguinte equação, já obtida por separação de variáveis:

$x \frac{X^{\prime \prime}{ }_{n}}{X_{n}}+\frac{X_{n}^{\prime}}{X_{n}}+x \frac{Y^{\prime \prime}{ }_{n}}{Y_{n}}=\frac{\rho}{E_{o}} \frac{\ddot{T}_{n}}{T_{n}}=-\alpha_{n}^{2}$

Para a resolução da função $v(x, y, t)$ iguala-se a equação (14) a uma constante $-\alpha_{n}^{2}$ negativa, pois espera-se que solução no tempo seja expressa em termos vibratórios, com senos e cossenos na resposta. Então, a EDO temporal fica:

$\frac{\rho}{E_{o}} \ddot{T}_{n}+T_{n} \alpha_{n}^{2}=0$ 
Com a aplicação da condição de velocidade inicial nula, a solução temporal fica na forma:

$T_{n}(t)=A_{n} \cos \left(\alpha_{n} t\right)$

Também se espera que ao longo da direção y haja um movimento em termos de senos e cossenos. Então, identifica-se o sinal do quociente da expressão envolvendo a variável $Y_{n}(y)$ como negativo, de modo que:

$Y_{n}^{\prime \prime}+Y_{n} \beta_{n}^{2}=0$

Então, em princípio, com a aplicação das condições de contorno, se teria $y_{n}(y)$ na forma:

$Y_{n}(y)=D_{n} \operatorname{sen}\left(\beta_{n} y\right)$

Mas, diferentemente dos problemas mais simples, não se pode deduzir imediatamente os valores de $\beta_{n}$ do argumento mostrado na equação anterior, pois tal restrição vai implicar numa EDO na variável $x$ inconsistente, conforme experiência efetuada. É preciso aguardar e resolver primeiramente a EDO em termos de $X_{n}(x)$.

Examinando-se, então, a equação diferencial $X_{n}(x)$, vê-se que sua forma é dada por:

$x X_{n}^{\prime \prime}+X_{n}^{\prime}+\left[\alpha_{n}^{2}-x\left(\beta_{n}\right)^{2}\right] X_{n}=0$

Esta não é uma equação trivial e sua solução não é encontrada facilmente nos livro clássicos de Matemática Avançada ou de Física Matemática [4]. Percebe-se que é um tipo especial da Equação de Laguerre. Segundo o software Wolfram Matemática 9.0, tal equação possui soluções do tipo:

$X_{n}(x)=e^{-x \beta_{n}}\left\{E_{n} U\left[-k, 1,2 x \beta_{n}\right]+F_{n} L\left[k, 2 x \beta_{n}\right]\right\}$

As funções base são as funções Hipergeométricas $U_{k} e$ os polinômios de Laguerre Lk. Na forma dada pela Eq. (20) já foram consideradas as ordens inteiras k para as funções base. Com a aplicação das condições de contorno, são geradas raízes que se relacionam com os autovalores temporais $\alpha_{n}$ e argumentos $\beta_{n}$ das autofunções referentes a variável y. Apenas valores de $\mathrm{k}$ maiores do que dois devem ser usados, pois é preciso que tais autofunções cruzem o eixo das coordenadas $x$ em pelo menos dois pontos.

A partir deste ponto, para a geração da solução completa $v(x, y, t)$, é preciso desenvolver termo a termo as diversas autofunções $X_{n}(x), Y_{n}(y)$ e $T_{n}(t)$ em função dos valores de $\alpha$ e $\beta$ calculados, seguindo as etapas comumente empregados em casos mais simples. No entanto, a implementação das integrais com as funções de Laguerre e Hipergeométrica exigem a utilização de um software adequado para a sua operacionalização.

\section{Conclusões}

Não foi propósito deste trabalho, tecer considerações detalhadas sobre as vantagens das principais técnicas voltadas para solução de EDPs. Certamente, existem categorias de problemas onde o desempenho de uma técnica é superior a das demais alternativas.

No problema específico que motivou esse trabalho, o MSV foi escolhido não só pelas suas qualidades, mas também pelas dificuldades operacionais envolvidas na aplicação de outras opções. Por exemplo, as Técnicas de Transformadas são instrumentos excelentes de solução, particularmente nos problemas de fronteira aberta e valor inicial, mas desde que as funções no espaço transformado sejam simples, o que não seria o caso aqui.

Entretanto, percebeu-se que a simplicidade apresentada pelo MSV nas aplicações mais clássicas deu lugar a grandes dificuldades conceituais e operacionais. Embora possa se cogitar que a aplicação de outras técnicas seja ainda mais proibitiva, a abordagem o problema proposto revestiu-se de grande complexidade e não pode prescindir do apoio de um software robusto para solução de muitos algebrismos difíceis, ratificando que o método de solução deve ser denominado rigorosamente de semi-analítico.

Algumas características do MSV se mantiveram: ao reduzir a EDP a um conjunto de EDOs, pode-se examinar o problema sob uma privilegiada ótica física, algo impossível quando se pensa numa solução por transformadas. Assim, o MSV continuou ostentando sua clareza, no sentido que suas etapas operacionais mantiveram a sistematização usualmente encontrada.

\section{Referências}

[1] E. Kreiszig, Matematica Superior, vol3, Livros Técnicos e Científicos Editora, Rio de Janeiro, 1976.

[2] G.STHEPHENSON, Uma Introdução as Equações Diferenciais Parciais.Edgar Blucher Ltda, São Paulo,1975.

[3] V.F. SILVA, Estratégias para Estudos de iluminação Sísmicas Baseadas na equação Acústica da Onda, Dissertação de Mestrado,Programa de Engenharia Civil, Universidade Federal do Rio de Janeiro, Rio de Janeiro,COPPE, 2011.

[4] E.JAHNKE, F. EMDE; Tables of Functions With Formulae and Curves, Dover Publ., New York, 1943. 